This document is published in:

Delgado Kloos, C. et al. (eds.) (2012). Towards Ubiquitous Learning: 6th European Conference of Technology Enhanced Learning, EC-TEL 2011, Palermo, Italy, September 20-23, 2011. Proceeding, (Lecture Notes in Computer Science, 6964), Springer, pp. 424-429.

DOI: 10.1007/978-3-642-23985-4_33

๑ 2011 Springer-Verlag Berlin Heidelberg 


\title{
Orchestration and Feedback in Lab Sessions: Improvements in Quick Feedback Provision
}

\author{
Israel Gutiérrez Rojas, Raquel M. Crespo García, Carlos Delgado Kloos \\ Universidad Carlos III de Madrid, Avda. Universidad 30, E-28911 Leganés, Spain
}

\{igrojas, rcrespo, cdk\} @it.uc3m.es

\begin{abstract}
The benefits of formative assessment and the provision of quick feedback to the learners are unquestionable. A lot of effort needs however to be devoted by the teacher in order to assess the learner's status and providing quality and valuable feedback when actually implementing these techniques in real-world situations during active learning activities. The aim of this work is to offer teachers a tool that facilitates the quick provision of relevant feedback to the students, implemented as backchannel notifications in a lab session for ensuring scalability. Although being a work-in-progress, the aforementioned tool has been partially evaluated in a real course on programming multimedia applications.
\end{abstract}

Keywords: formative feedback, backchannel notifications, orchestration.

\section{Introduction}

Active learning is nowadays perceived as an engaging and effective learning methodology. When applied into actual class it can however be hindered by both intrinsic as well as circumstantial drawbacks. This work is inspired by the experience in an actual active learning-oriented course on Multimedia Applications. Although being addressed to $3^{\text {rd }}$ year students, one particularly critical handicap in this course is students' reticence -active learning demands a bigger effort than just attending lecturers-, asking for step-to-step guidance by the teachers rather than willingly make an effort to solve the problems themselves and asking just for punctual help. This leads to scalability problems that jeopardize the feasibility of the methodology. Thus there is a need of better orchestration for the teachers to maximize the quality and efficiency of the interactions with the students in lab sessions.

Two ways are used for providing feedback to the students in the lab: personalized feedback directly delivered to individuals or teams; or explanations to the whole audience, intended to be used for common issues, general remarks and comments. The first type of interaction is highly effective (mainly due to the undivided attention provided by the students and its personalized nature), but not so efficient depending on the size of the audience. The second one is much more efficient as all the students are addressed simultaneously; its effectiveness will however depend on several aspects, among other, the attention devoted by the students and the relation of the explanation to the actual student work until that moment. 
The objective of this work is to provide a supporting system for helping the teacher to scaffold the students during active learning hands-on sessions. The underlying idea is to provide a feedback mechanism that, while remaining efficient (addressing all the students) is more effective than generic explanations and is delivered to the students at the correct moment in time.

This work is founded on three main research lines:

- Use of communication backchannel (alternative communication channel supported by telematics) in class

- Benefits of the provision of quick formative feedback to the students

- Learning orchestration (a metaphor used to address the challenges of coordinating the complexity of conducting learning activities)

\section{Related Work}

Using backchannels for generating instant feedback within lectures is reported as a factor for potential success according to [2]. This is consistent with [1] who notes that: "...the specific ways in which they can influence teaching pedagogy and learning opportunities are less well understood." Although backchannels are typically used for bidirectional communication, the potential and learning opportunities are expected to be applicable to one-way notifications too.

Regarding pedagogical trends, the main goal of formative assessment is to provide feedback to the learner in order to improve his/her learning; thus the growing importance it has been receiving in the last decades [4]. Additionally, it promotes the continuous tracking of the learner's achievements. As explained in [5], formative feedback "helps learners become aware of any gaps that exist between their desired goal and their current knowledge, understanding, or skill and guides them through actions necessary to obtain the goal".

Finally, different works report the benefits of appropriate learning orchestration. In particular, a distributed awareness system based on lamps (Lantern) that improves the orchestration of the class is presented in [3]. In our work, although the system is not distributed in the classroom it provides contextual information about the physical distribution of the room; hence, it preserves the fast decision making with a glance but adding more information obtained from the students' events monitoring.

\section{Proposed Solution}

The proposed solution is based on two artifacts: backchannel notifications (for quick feedback provision) together with an application for supporting orchestration.

Backchannel notifications are implemented using HTML5 technologies: web sockets, which permit real-time interaction between clients (browsers) and web notifications, which allow sending simple messages to the foreground of the clients' desktop. Combining both technologies a backchannel notification tool has been implemented, so that: 
- When the students access to the wording of a lab session, they have the possibility to subscribe to the notifications channel using a "Connect" button in the same page of the wording.

- The teachers of the lab sessions are provided with a web interface used to send notifications to the subscribed students. This allows the teacher to provide feedback to the students as demanded according to the evolution observed in the class, as usually there are common doubts shared by the majority of students.

- Notifications are immediately shown in the foreground student screen and remain visible until the student chooses to close them.

The notifications application is being integrated in a higher level, more global, orchestration and feedback system. This system aims at providing relevant and efficient information to the teachers that allow them to improve the orchestration of the session. This awareness information is related to the performance of the students in the session as well as the required and provided teacher's support, and can be used by teachers to decide the most appropriate way of providing efficient and accurate feedback to the students.

The student interface is designed to be useful and easy to use in order to remain non-intrusive. It is integrated in the problem statement (wording) of the lab session (provided as a web page) and consists on a progress indicator including:

- a button to indicate student(s) have started working on this section

- a button to ask for teacher help

- a button to indicate the finalization of the section

- the expected time for the student to complete the exercise (on the right)

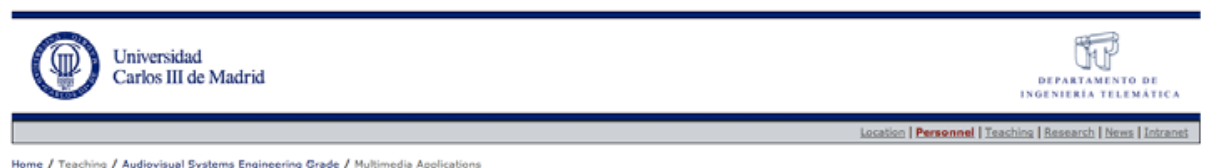

\section{Processors in JMF: Creation of Time-lapses}

\section{(1) (?)}

\section{Section 1}

According to [1], Time-lapse photography is a cinematography technique whereby the frequency at which film frames are captured (the frame rate) is much lower than that which will be used to play the sequence back. When replayed at normal speed, time appears to be moving faster and thus lapsing. For example, an image of a scene may be captured once every second, and then played back at 30 frames per second; the result would be an apparent increase of speed by 30 times. Time-lapse photography can be considered to be the opposite of high speed photography.

Processes that would normally appear subtle to the human eye, such as the motion of the sun and stars in the sky, become very pronounced. Time-lapse is the extreme version of the cinematography technique of undercranking, and can be confused with stop motion animation.

Fig. 1. Student interface

Teachers interface (Figure 2) has been designed for mobile devices that can be easily operated at the lab, mainly tablet PCs though smartphones could also be used. The tool summarizes awareness information over the map of the lab workstations: 
- Students' progress: indicated as the number of the exercise they are currently working on, in the upper part of each computer icon.

- Students asking for help: background color of the upper part of the computer icon, ranging from orange to red as time passes.

- Students with slow rate progress: computer icon stroke turns orange for pointing out students delayed with respect to the main group.

- Tutoring time already consumed: shown in the lower part of the computer icon.

- Consumed tutoring time relative to the most tutored student: the bottom part of the computer icon is a progress bar colored in blue.

- Surrounded computers represent the students being assisted (blue) and the next ones to be tutored (the ones with highest waiting time, in red)

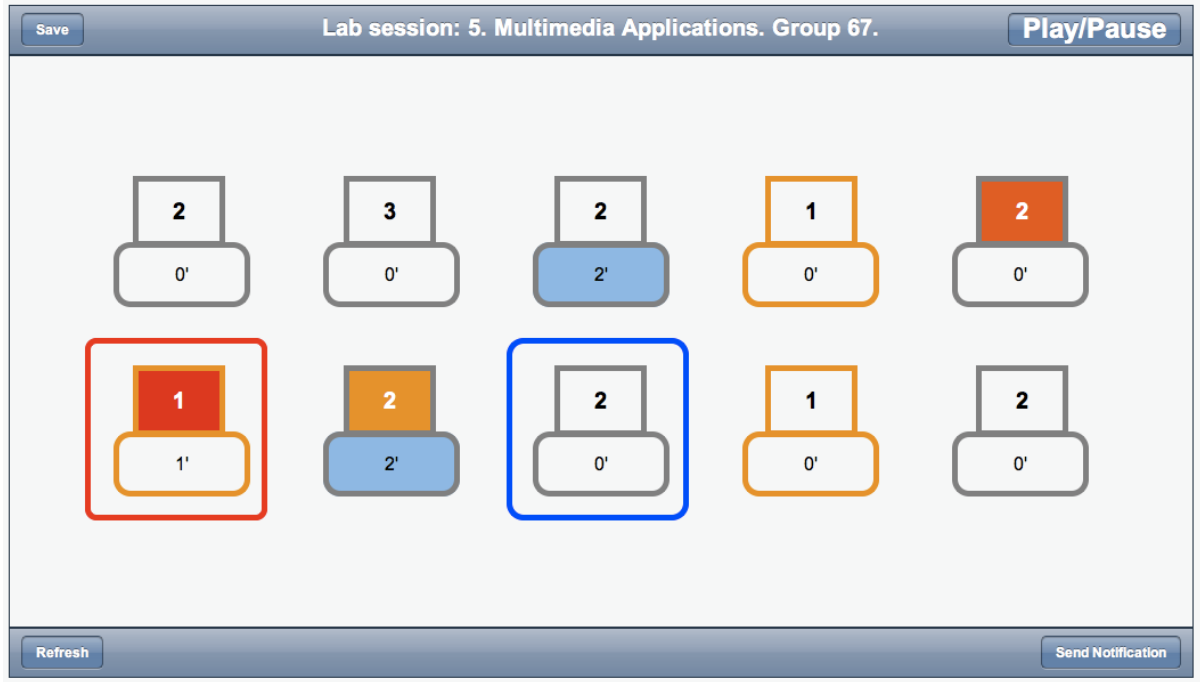

Fig. 2. Orchestration and feedback application interface

Any time, the teacher can catch a glimpse of the current progress of the class and the groups asking for help during the session. At a glance, (s)he can easily decide the next group of students to tutor, applying his/her own criteria. For example, higher priority can be given to either the students that have been waiting more time or to the students stuck at one of the first exercises (that is, delayed from the rest of the class), and also take into account the time already spent with each group. Once decided the group to assist, just touching the group will allow the system to track information about the time devoted to each group. If one problem is relevant for more students, and the progress state shows that it is the appropriate time, the notifications system can be used for making all the students aware of this relevant information. Having finished the tutoring action, the teacher decides the next group to attend or, if nobody is waiting for help, stop the timer that was measuring the time spent with the tutored group using the Play/Pause button in Fig. 2. 
Following this workflow, several types of information will be stored in the system that will allow the teacher to reflect about the effectiveness of the session:

- Tutoring time, meaning time devoted in the session to solve doubts of the students personally, both for each student as the aggregate measure for the class.

- Performance of the students in the session, both individual and aggregated.

- Typical problems posed in this session (from submitted notifications).

\section{Classroom Experience}

The feedback and orchestration tool was used in the context of a Multimedia Applications course during two lab sessions. The students had to acquire some knowledge about HTML5, concretely the use of the 2D canvas and the drag\&drop interaction, developing some examples in Google Chrome. A button was incorporated in the wording for connecting to the notifications services. This channel was used by the teachers to provide some feedback to the students at class level, without interrupting their work and disrupting their attention. Being a work in progress, only the notifications have been experimentally tested in the class.

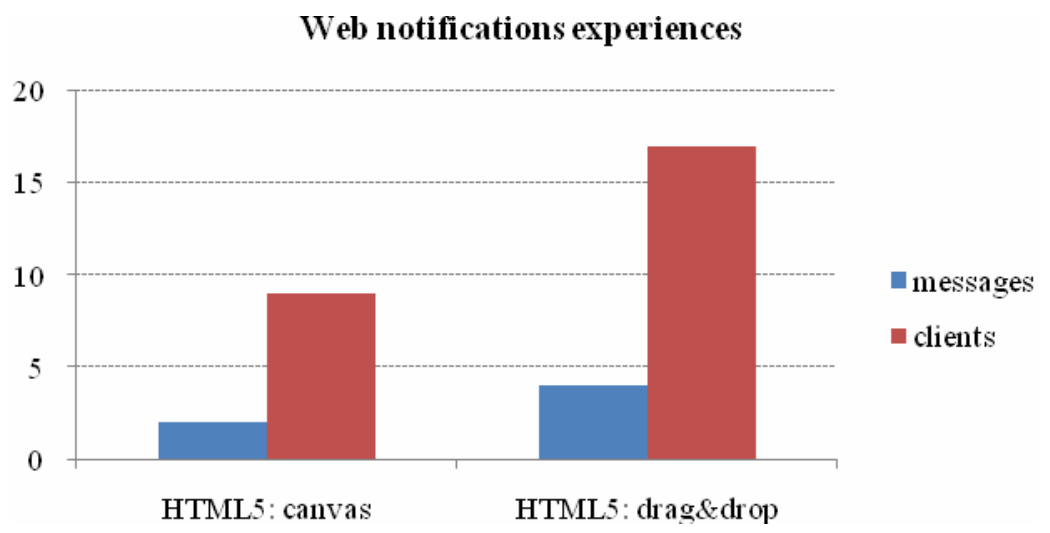

Fig. 3. Number of clients and messages sent in two experiences of websocket notifications

Figure 3 summarizes the use of notifications along the two sessions. In the first one, two notifications were sent and only 9 teams of 2 students each (18 students out of 40 in the class) connected to the notifications channel and received the feedback. In the second session, more feedback (broadcasted messages) was necessary (4 messages) as the drag\&drop interaction in HTML5 is an advanced aspect. In this case, more students (17 groups, i.e. 34 students out of 40) connected to the server. There is an increasing trend in the addressed students (9 to 17) and the notifications sent (2 to 4 ), as users -both teachers and students- got familiar with the system and the needs for feedback increased. 


\section{Conclusions and Future Work}

This paper presents a work-in-progress tool for lab sessions orchestration and provision of effective quick feedback to the participant students. The system usefulness is grounded by the state-of-the-art review and the fact that meets actual requirements by the course teachers. Apart from taking into account pedagogical aspects like orchestration and formative feedback, it also allows the collection of data useful for students assessment and quality improvement. It allows the teacher to be more aware of the problem-solving process followed by the students, and students can be assessed based on this process besides the product they deliver.

As future works in the orchestration and notification tool we have spotted two main lines of work. Regarding experimental validation, further evaluation of the expected benefits and utility of the system is to be done. Regarding application development, some improvements are being considered:

- Defer the delivery of notifications to the students depending on their progress in the activity, according to the information provided through the orchestration system; the underlying intention being to avoid diverting the students' attention.

- Create a visualization of the notifications flow; it will be much related to the difficulties that the students are encountering.

- Long term, create an intelligent tutoring system that sends feedback automatically to the students based on their progress in the lab session, their performance in previous exercises and common mistakes of the students.

Acknowledgments. This research has been partially supported by the project "Learn3: Towards Learning of the Third Kind" (TIN2008-05163/TSI) of the Spanish "Plan Nacional de I+D+i" and the Madrid regional project "eMadrid: Investigación y Desarrollo de tecnologías para el e-learning en la Comunidad de Madrid" (S2009/TIC-1650).

\section{References}

1. Yardi, S.: Whispers in the Classroom. In: McPherson, T. (ed.) Digital Youth, Innovation, and the Unexpected. The John D. and Catherine T. MacArthur Foundation Series on Digital Media and Learning, pp. 143-164. The MIT Press, Cambridge (2008)

2. Conole, G., Alevizou, P.: A literature review of the use of Web 2.0 tools in Higher Education. A report commissioned by the Higher Education Academy (2010)

3. Alavi, H.S., Dillenbourg, P., Kaplan, F.: Distributed awareness for class orchestration. In: Cress, U., Dimitrova, V., Specht, M. (eds.) EC-TEL 2009. LNCS, vol. 5794, pp. 211-225. Springer, Heidelberg (2009)

4. Sadler, D.R.: Formative assessment and the design of instructional systems. Instructional Science 18(2), 119-144 (1989), doi:10.1007/BF00117714.

5. Boston, C.: The concept of formative assessment. Practical Assessment, Research \& Evaluation 8(9) (2002),

http: / / PAREonline. net/getvn. asp?v=8\&n=9 